\title{
Prestack depth migration using variable large-step
}

\author{
Yang Qiqiang \\ Department of Mathematics, Qiongzhou college, Sanya, China \\ keeponmoving@126.com
}

Keywords: variable large-step, prestack depth migration, FFD, synthesized areal shot-record migration.

\begin{abstract}
A variable large-step prestack depth extrapolation migration method is presented. In the extrapolation process adopting a large depth step can keep efficiency but at a cost of low accuracy. However, if the depth step is allowed to vary we can use a relative small depth step for accuracy where required and large depth step where possible to retain efficiency. The intermediate depth samples are obtained by applying phase-shift operator and interpolations. The Fourier finite-difference (FFD) operator is employed as the depth extrapolation operator. The variable large step method is also suitable to other extrapolation operators such as the split-step Fourier (SSF) operator and the phase-shift plus interpolation (PSPI) operator. To make this approach more efficient and economical, the method is applied to the synthesized areal shot-record migration. Two numerical examples have been used to assess the feasibility of this approach.
\end{abstract}

\section{Introduction}

As so far, prestack depth migration is the most efficient method for imaging complex structures. The wave equation prestack depth migration methods based on the wave extrapolations has got more and more realization in practical production in recent years. However, the methods are computationally expensive because the migration process involves a huge amount of computations required for the extrapolation process of the prestack data.

The proposition of the synthesized areal shot-record migration [1] has improved the efficiency of the wave equation prestack depth migration. In the approach the point shot records are synthesized into an areal source response prior to shot-record migration, and subsequently an enormous data reduction can be obtained. The resulting areal shot record can be migrated using the same scheme as the one for conventional shot-record migration. The main advantage of the areal shot-record migration is that the extrapolations need not be done for all the individual shot records, but for the areal shot record only.

A straight-forward approach to implementing prestack depth migration involves the use of a fixed depth step. While attractive for its simplicity, a fixed depth step can not take advantage of neither the depth varying frequency content, nor the increase in velocity with depth, usually found in seismic data. However, if the depth step is allowed to vary, the features may be utilized.

Adopting large depth step is a straightforward way to improve the efficiency of prestack depth migration methods based on wave extrapolation. But the accuracy is influenced and the qualities of imaging go worse as the depth step increases. In many finite difference and finite element methods outside the seismic industry varying grid spacing and indeed adaptive curvilinear grids are commonplace. In the seismic industry these adaptive approaches are less common. Jastram and Behle [2] investigated varying the grid spacing for acoustic wavefield modeling and Mufti. In this paper, the author proposed a variable large depth step method which produces accurate migration images with high efficiency. The Fourier finite-difference operator [3] is employed as the depth extrapolation operator. The variable large step method is also suitable to other extrapolation operators such as the split-step Fourier operator [4] and the phase-shift plus interpolation operator [5]. In migration examples, we first use a 2-D slice of the SEG/EAGE 3-D salt model to test the method. A field data is then used to demonstrate the feasibility and efficiency of the method. 


\section{Migration with large-step wavefield extrapolation}

In the migration methods based on wave field depth extrapolation, it is easy to notice that the wave fields in different depths are similar except the phase. This suggests that we can utilize the phase-shift formula to deal with the intermediate wave fields during wave field extrapolations. The 2-D phase-shift formula[6] reads:

$$
p\left(z+\Delta z, k_{x}, \omega\right)=p\left(z, k_{x}, \omega\right) \exp \left(i \theta_{z} \Delta z\right)
$$

where

$$
\theta_{z}= \pm \frac{\omega}{v} \sqrt{1-\frac{k_{x}^{2} v^{2}}{4 \omega^{2}}},
$$

$k_{x}$ and $\omega$ are the Fourier counterparts of the horizontal coordinate $x$ and time $t, z$ represents the vertical coordinate, $p(z, x, t)$ represents the wave field. The sign of $\theta_{z}$ can be differentiated as follows. In a downward extrapolation process, i.e., when $\Delta z$ in Eq.1 is positive, sign agreement between $\theta_{z}$ and $\omega$ corresponds to waves that move in the negative $t$ direction. On the other hand, when $\theta_{z}$ and $\omega$ have opposite signs, Eq.1 represents waves that movie in the positive $t$ direction. The downward extrapolation of recorded seismic data is an inverse process, we are interested in

$$
\theta_{z}=\frac{\omega}{v} \sqrt{1-\frac{k_{x}^{2} v^{2}}{4 \omega^{2}}}
$$

And in the downward extrapolation of source waves, we are interested in

$$
\theta_{z}=-\frac{\omega}{v} \sqrt{1-\frac{k_{x}^{2} v^{2}}{4 \omega^{2}}} \text {. }
$$

In Eq.3, let $k_{x}=0$, then the 2-D phase-shift equation is simplified to the 1-D phase-shift equation:

$$
p(z+\Delta z, \omega)=p(z) \exp \left(i \frac{\omega}{v}\right) \text {. }
$$

Obviously,Eq.5 satisfies the one-way wave equation:

$$
\frac{\partial p(z, \omega)}{\partial z}=i \frac{\omega}{v} p(z, \omega) \text {. }
$$

We first obtain the wave field $P(z+N \Delta z, x, \omega)$ from the known wave field $P(z, x, \omega)$ by large-step wave field extrapolation. We just consider the change of the phase, and then the wave filed from depth $z$ to $z+n \Delta z$ can be obtained by extrapolating the wave field $P(z, x, \omega)$ downward:

$$
P_{1}(z+n \Delta z, x, \omega)=P(z, x, \omega) \exp \left(i \frac{\omega}{v(x)} n \Delta z\right) .
$$

In like manner, the wave filed from depth $z$ to $z+n \Delta z$ can also be obtained by extrapolating the wave field $P(z+N \Delta z, x, \omega)$ upward:

$$
P_{2}(z+n \Delta z, x, \omega)=P(z+N \Delta z, x, \omega) \exp \left(i \frac{\omega}{v(x)}(n-N) \Delta z\right) .
$$

Finally, we perform linear interpolation on the wave field $P(z, x, \omega)$ and $P(z+n \Delta z, x, \omega)$ to get the wave field in depth $z+n \Delta z$ as follows:

$$
P(z+n \Delta z, x, \omega)=\left(\frac{N-n}{N}\right) P_{1}(z, x, \omega)+\left(\frac{n}{N}\right) P_{2}(z+N \Delta z, x, \omega) .
$$

A similar scheme can be used to calculate the source waves adopting Eq.3. Now a crucial issue is how to get an appropriate $N$. If $N$ is too large, imaging problems may occur. We can use a depth step dependent on frequency and velocity:

$$
\delta_{z}=\frac{v}{2 f},
$$


where $\delta_{z}$ is the depth step, $v$ is the local velocity and $f$ is the current frequency in Hertz. In a migration that is able to handle lateral velocity variations, the above conditions can not be satisfied easily because it would imply a laterally varying depth step. This could be solved by choosing the minimum velocity $v_{\min }$ in each depth slice. To represent all migrated frequencies up to the Nyquist frequency, we set $f$ to be the maximum migrated frequency $f_{\max }$. Consequently, the value of $N$ is determined by

$$
N=\mathfrak{R}\left(\frac{v_{\min }}{2 \Delta z^{*} f_{\max }}\right) .
$$

where $\mathfrak{R}(x)$ represents the maximum integer that is not bigger than the real parameter $x . \Delta z$ is selected according to velocity samples and the minimum value of $\delta_{z}$ shown in Eq.10.

\section{Example}

To demonstrate the feasibility and efficiency of the method, we have tested the method for the synthesized areal shot-record migration with Fourier Finite-difference operator using the 2-D SEG/EAGE salt model and the Baicheng (XinJIang, China) field data.

The migrated sections for the salt model are depicted in Fig.1. Depth and distance are measured in kilo feet. The plane waves are synthesized on the surface and the incidence angles of the waves are from $-45^{\circ}$ to $+45^{\circ}$ with interval $1^{\circ} . \Delta z$ is set to be 20 feet. From the figure we see that if the depth step is too large, the qualities of imaging can not be guaranteed. Migration using variable large extrapolation steps provides comparative migration images to that using fixed small depth steps in a computationally efficient way.

Fig. 2 provides two migration images that demonstrate the approach applied on the Baicheng field data. Depth and distance are measured in kilometers. The plane waves are also synthesized on the surface and the incidence angles of the waves are from $-40^{\circ}$ to $+40^{\circ}$ with interval $0.5^{\circ}$. The figure shows that the variable depth step method produces accurate result with much less extrapolations.

\section{Summary}

The preceding figures demonstrate the validity of the variable large-step migration method. Using a fixed depth step either can not guarantee the accuracy of imaging or is computationally expensive. On the contrary, adopting a variable depth step would retain the accuracy and improve the efficiency where possible. We have presented a method for the selection of appropriate depth step. The variable large depth step migration method is now an efficient, accurate and economical migration approach.

\section{Acknowledgements}

This research was supported by Hainan Natural Science Foundation (Grant No. 113008).

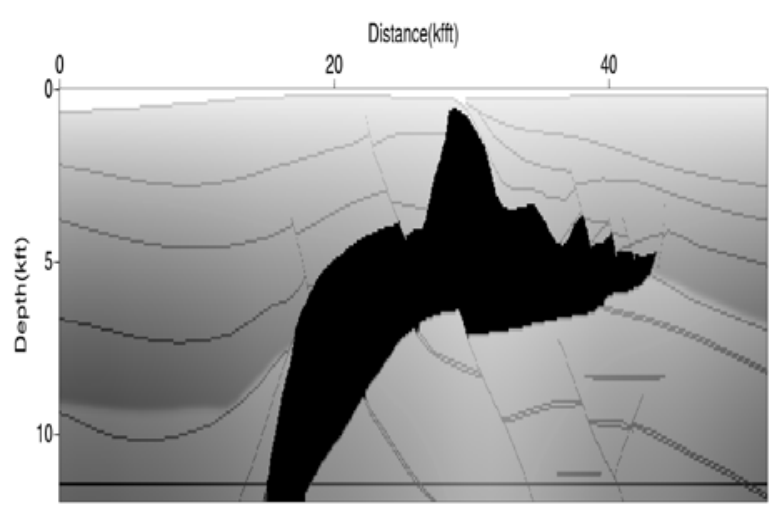

a

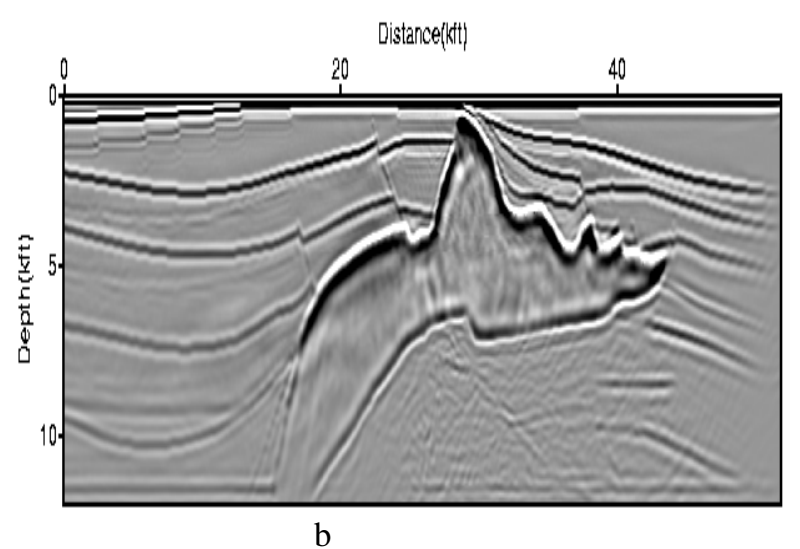

b 


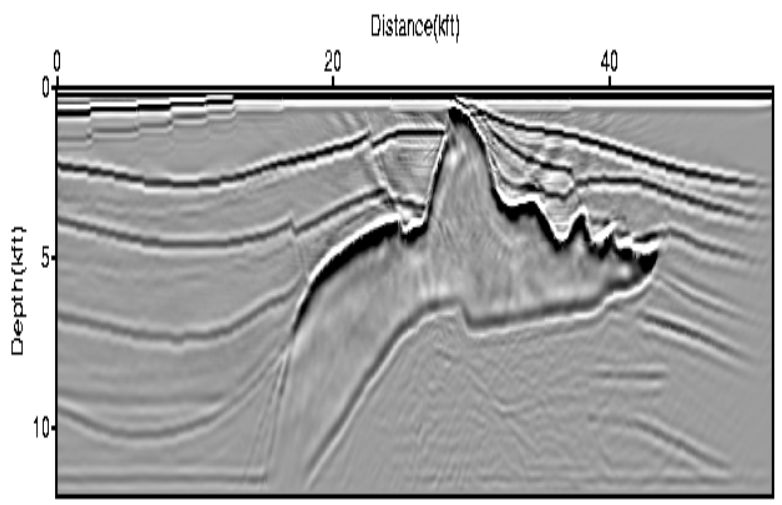

C

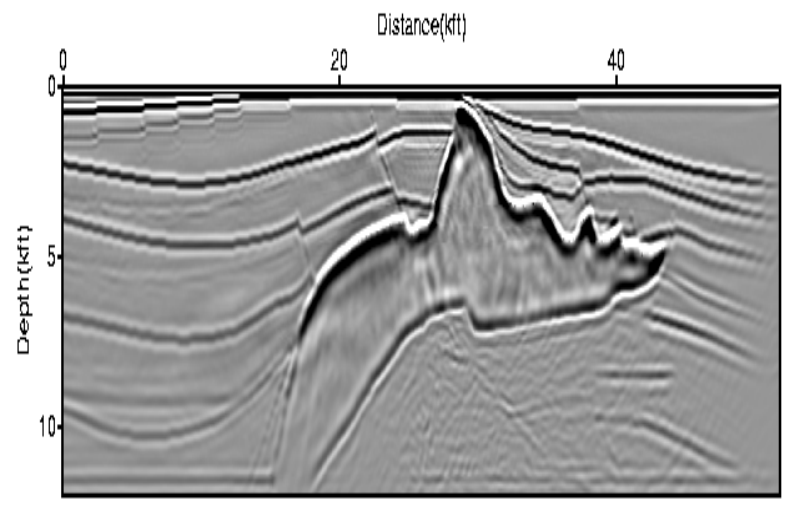

d

FiG. 1. Test for the large-step extrapolation method using the SEG AA' data set. (A) Profile A-A ' from the SEG/EAGE salt model. Darker shades denote higher velocities. (B) Migration using $f$ extrapolation step 20 feet. (C) Migration using depth step 160 feet. (D) Migration using variable large depth step.

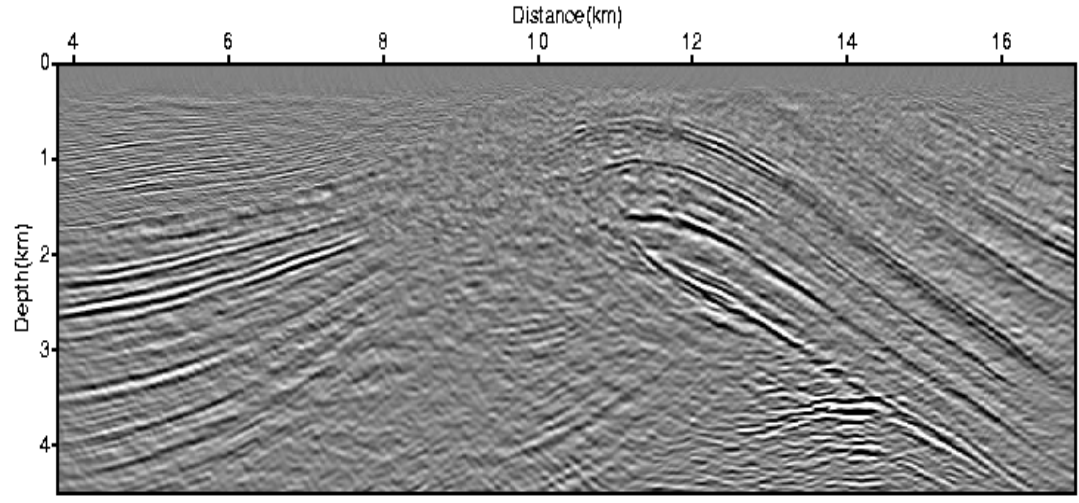

e

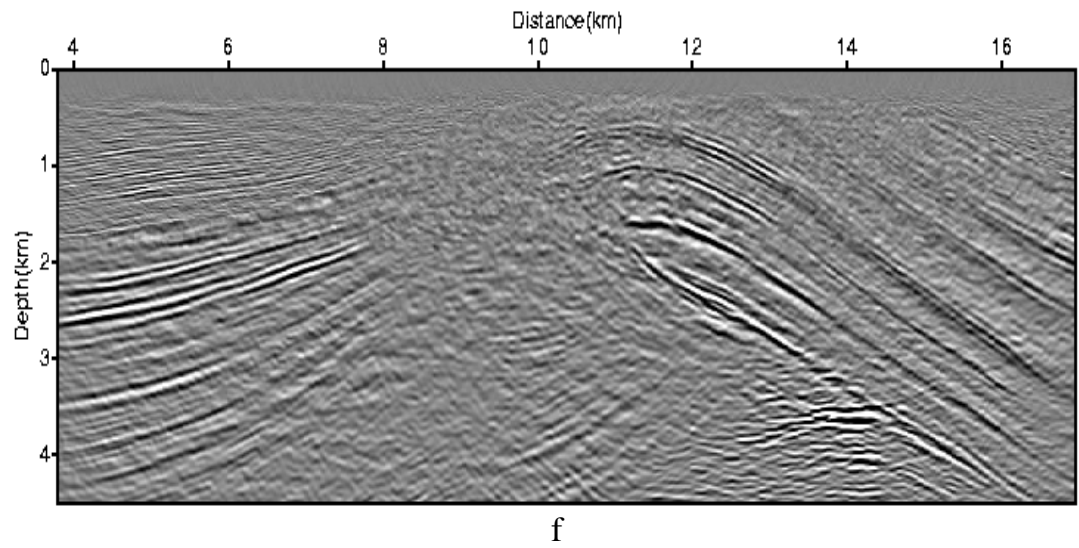

FiG. 2. Test using the Bairen (Xinjiang, China) field data. (A) Migration using extrapolation step 5 m. (B) Migration using variable depth step.

\section{References}

[1] Berkhout,A.J., Areal shot record technology:J.Seis.Expl.,1,no.3.(1992)

[2] Jastram, C. \& Behle, A, Acoustic modeling on a grid of vertically varying spacing, Geophys. Psosp., 40 (1992), 157 169.

[3] Dietrich Ristow and Thomas Ruhl, Fourier Finite-difference migration, Geophysics, 59 (1994), 1882-1893.

[4] Stoffa P L, Fokkema J T, de Luna Freire R M et al. Split-step Fourier migration. Geophysics, 55(4) (1990),410-421.

[5] Gazdag J., Squazzero P., Migration of seismic data by phase shift plus interpolation, Geophysics 49 (1984),124-131. 
[6] Gazdag, J., Wave equation migration with the phase-shift method, Geophysics, 43 (1978), 1342-1351. 